Pacific Journal of Mathematic 


\title{
ON THE GEOMETRY OF COMBINATORIAL MANIFOLDS
}

\author{
Michael A. PenNa
}

On a smooth manifold there are classical relations between vector fields and derivations of the smooth function algebra, and between differential forms and alternating linear maps of vector field tuples. In this paper similar relations are obtained for combinatorial manifolds. As an application of these results the existence of connexions and parallel translation on combinatorial manifolds is established.

0. Introduction. The basic theme of (6) and (7) is that there is a striking similarity between the geometry of smoth manifolds and the geometry of simplicial complexes. The purpose of this paper is to continue this theme for smooth manifolds and combinatorial manifolds. (Note: Henceforth a combinatorial $n$-manifold $M$ is the geometric realization of a simplicial complex for which the closed star of each point can be mapped by a homeomorphism onto a combinatorial $n$-ball in $R^{n}$ in such a manner that each simplex of $M$ is mapped affinely to a simplex in $R^{n}$. Furthermore all combinatorial manifolds are assumed to have no boundary. See (1) and (9) for related definitions.)

Section 1 is devoted to a brief review of some of the terminology and results of (6) and (7). The goal of $\S 2$ is the characterization of continuous vector fields on combinatorial manifolds. The main technical results of this paper are proved in $\S 3$; these results are complied in the following statement.

THEOREM. Let $M$ be a combinatorial n-manifold, $A(M)$ the ring of piecewise smooth real-valued functions on $M, \mathscr{P}(M)$ the $A(M)$ module of continuous vector fields on $M$, and $E(M)$ the $A(M)$-module of piecewise smooth 1-forms on $M$. Then:

(1) there is an $A(M)$-module isomorphism between $\mathscr{X}(M)$ and the module $\mathscr{D}(M)$ of derivations of $A(M)$; consequently $\mathscr{E}(M)$ is a Lie algebra over $R$ with respect to

$$
[X, Y] f=X(Y f)-Y(X f)
$$

for $X, Y \in \mathscr{P}(M)$ and $f \in A(M)$,

(2) there is an $A(M)$-module isomorphism between $E(M)$ and the module $\mathrm{Hom}_{A(M)}(\mathscr{X}(M), A(M))$ of $A(M)$-linear maps from $\mathscr{X}(M)$ to $A(M)$,

(3) there is an $A(M)$-module isomorphism between $\Lambda^{q} E(M)$ 
and the module $\mathrm{Alt}_{A(M)}\left(\mathbf{X}^{q} \mathscr{X}(M), A(M)\right)$ of $A(M)$-linear alternating maps from $\mathrm{X}^{q} \mathscr{P}(M)$ to $A(M)$, and

(4) if $d: \Lambda^{*} E(M) \rightarrow \Lambda^{*} E(M)$ is the differential of the de Rham complex of $M$ and $\theta \in \Lambda^{q} E(M)$, then $d \theta \in \Lambda^{q+1} E(M)$ is given by the formula

$$
\begin{aligned}
d \theta\left(X_{1}, \cdots, X_{q+1}\right)= & \sum_{j}(-1)^{j+1} X_{j} \theta\left(X_{1}, \cdots, \hat{X}_{j}, \cdots, X_{q+1}\right) \\
& +\sum_{i<j}(-1)^{i+j} \theta\left(\left[X_{i}, X_{j}\right], X_{i}, \cdots, \hat{X}_{1}, \cdots, \hat{X}_{j}, \cdots, X_{q+1}\right)
\end{aligned}
$$

for $X_{1}, \cdots, X_{q+1} \in \mathscr{E}(M)$.

In $\S 4$ these results are used to show that connexions exist on combinatorial manifolds, and that such connexions can be described (or defined equivalently in terms of differential forms (Cartan connexions), vector fields (Koszul connexions), or compatible collections of connexions defined on individual simplices. Finally, such connexions are interpreted geometrically in terms of parallel translation.

The main results of this paper are motivated by analogous smooth results; these smooth analogs are presented in (2) and (8), for example. Results similar to some of those presented in $\S 3$ appear in (3); these results, however, are obtained from a much different point of view. The present work is motivated by related work of Professor Howard Osborn (see [4] and [5]); I am deeply indebted to him for all he taught me.

1. Review. In this section, and throughout the sequel, simplicial complexes will be locally finite and finite dimensional. A simplicial complex is understood to be a space $K$ together with a fixed triangulation of $K$ by simplices.

Let $K$ be a simplicial complex. A small open neighborhood $U$ of $x_{0} \in K$ is the intersection of the open star of $x_{0}$ with any other open neighborhood of $x_{0}$. The intersection $U_{\alpha}$ of a small open neighborhood $U$ of $x_{0}$ with any simplex $\sigma_{\alpha} \subseteq K$ which contains $x_{0}$ is a wedge of $U$. Each such wedge $U_{\alpha}$ is affinely homeomorphic to an open subset $V_{\alpha}$ of

$$
s^{n}=\left\{x=\left(x_{i}\right) \in R^{n} \mid x_{i} \geqq 0 \forall i\right\},
$$

where $n=n_{\alpha}=\operatorname{dim} \sigma_{\alpha} ;$ a map $\psi_{\alpha}: U_{\alpha} \rightarrow V_{\alpha} \subseteq s^{n}$ establishing this homeomorphism is a simplicial chart. A small open subset $U \subseteq K$ is a subset which is a small open neighborhood of some point $x_{0} \in K$. The set of small open subsets of $K$ forms a basis for the topology of $K$. 
A piccewise smooth real-valued function $f$ defined on a small open subset $U$ is a continuous function $f: U \rightarrow R$ whose restriction to each wedge $U_{\alpha}$ of $U$ is smooth. If $U$ is a small open neighborhood of $x_{0}$, a coordinate system on $U$ with origin $x_{0}$ is a collection $u=$ $\left\{u_{i}: U \rightarrow R\right\}$ of piecewise smooth real-valued functions defined on $U$ such that for each wedge $U_{\alpha}$ of $U$ and any simplicial chart $\psi_{\alpha}: U_{\alpha} \rightarrow V_{\alpha}$,

$$
\left\{u_{i} \circ \psi_{\alpha}^{-1}: V_{\alpha} \longrightarrow R \mid u_{i} \in u \text { and }\left.u_{i}\right|_{C_{\alpha}} \neq 0\right\}
$$

is a coordinate system on $V_{\alpha}$ with origin $\psi_{\alpha}\left(x_{0}\right)$. For each $u_{i} \in u$ we let St $u_{i}$ denote the support of $u_{i}$.

The rules for change of coordinates are:

(1) If $u$ is a coordinate system on $U$ with origin $x_{0}$ and $W \subseteq U$ is a small open neighborhood of $x_{1} \in U$, then there is an induced coordinate system on $W$ with origin $x_{1}$ given by

$$
w=\left\{u_{i}-u_{i}\left(x_{1}\right): W \longrightarrow R \mid u_{i} \in u \quad \text { and }\left.\quad u_{i}\right|_{W} \neq 0\right\}
$$

where $u_{i}\left(x_{1}\right)$ is a constant function.

(2) If $u$ and $w$ are coordinate systems on $U$ with origin $x_{0}$ then there are real constants $c_{i j}$ for which $u_{i}=\sum_{j} c_{i j} w_{j}$ for each $u_{i} \in u$ where $w_{j} \in w$. In fact if the first $p$ functions of both $u$ and $w$ coordinatize the $p$-simplex in whose interior $x_{0}$ is contained, then the matrix $\left(c_{i j}\right)$ is of the form

$$
\left(c_{i j}\right)=\left(\begin{array}{c|c}
\begin{array}{c}
\text { invertible } \\
p \times p \text { matrix }
\end{array} & 0 \\
\hline * & \begin{array}{l}
\text { invertible } \\
\text { diagonal } \\
\text { matrix }
\end{array}
\end{array}\right) .
$$

There is a category, called the category of simplicial bundles, and a functor defined on the category of simplicial complexes with values in the category of simplicial bundles, called the tangent functor, which associates to each simplicial complex $K$ a tangent bundle $\tau(K): T K \rightarrow K$.

For example, the tangent bundle $\tau(K): T K \rightarrow K$ of the simplicial complex $K$ obtained by pasting two 1 -simplices together at a vertex is illustrated in Diagram 1. Observe that in this example fibers are vector spaces, but $\tau(K)$ is not a vector bundle since fiber dimensions may vary from point to point. This bundle is the canonical example of a simplicial bundle, and the property of varying fiber dimensions is characteristic of simplicial bundles. 


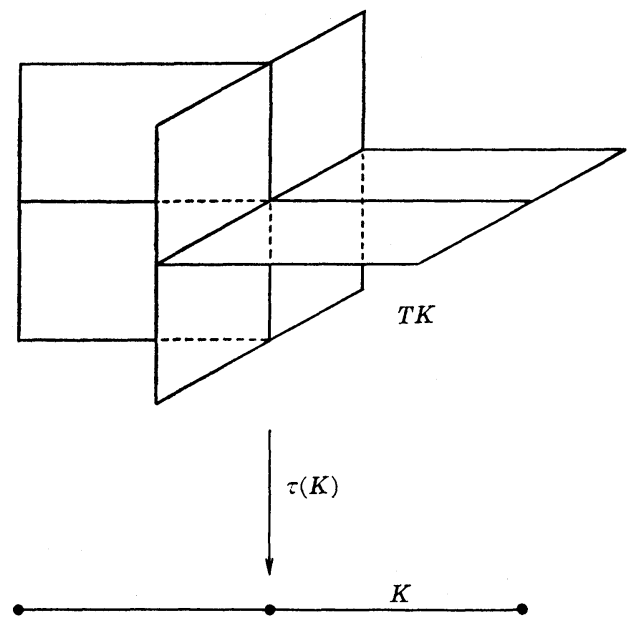

DIAGRAM 1

Tangent bundles are constructed as follows:

First let $U \subseteq K$ be a small open subset and $u$ a coordinate system on $U$ with origin $x_{0}$. Let $V(u)$ be the real vector space with basis $\left\{\left(\partial / \partial u_{i}\right)_{x_{0}} \mid u_{i} \in u\right\}$, and let $T(U ; u)$ be the subspace of $U \times V(u)$ consisting of all $\left(x, \sum_{i} c_{i}\left(\partial / \partial u_{i}\right)_{x_{0}}\right)$ such that $c_{i}$ is nonzero only if there is a wedge $U_{\alpha} \subseteq U$ for which $x \in U_{\alpha}$ and $\left.u_{i}\right|_{U_{\alpha}}$ is a coordinate function on $U_{\alpha}$. Projection onto the first factor gives a map $\tau(U ; u): T(U ; u) \rightarrow U$.

Next let $T K=\Perp T(U ; u) / \sim$, the free union of $T(U ; u)$ over all possible combinations of $U$ and $u$ modulo the equivalence relation $\sim$ : For $\left(x_{0}, v_{0}\right) \in T\left(U_{0} ; u_{0}\right)$ and $\left(x_{1}, v_{1}\right) \in T\left(U_{1} ; u_{1}\right),\left(x_{0}, v_{0}\right) \sim\left(x_{1}, v_{1}\right)$ iff $x_{0}=x_{1}$ and, after inducing coordinate systems $w_{0}$ and $w_{1}$ on a small open neighborhood $W$ of $x_{0}=x_{1}, v_{1}=\sum_{j} c_{j}\left(\partial / \partial w_{j}\right)$ for $w_{j} \in w_{1} \Rightarrow v_{0}=$ $\sum_{i j} c_{j} c_{i j}\left(\partial / \partial w_{i}\right)$ for $w_{i} \in w_{0}$ where $\left(c_{i j}\right)$ is the transition matrix from $w_{0}$ to $w_{1}$. There is a map $\tau(K): T K \rightarrow K$ induced by the maps $\tau(U ; u)$, and this is the tangent bundle of $K$.

2. Continuous vector fields. A vector field on the simplicial complex $K$ is a function (not necessarily a continuous function) $X: K \rightarrow T K$ for which $\tau(K) \circ X=\mathrm{id}_{K}$ and which satisfies a certain piecewise smoothness condition (see [7]). We are now interested in studying continuous vector fields $X: K \rightarrow T K$. Under the assumption of continuity, the piecewise smoothness of a vector field $X$ may be described as follows.

DEFINITION 2.1. A continuous vector field on the simplicial complex $K$ is a (continuous) map $X: K \rightarrow T K$ for which $\tau(K) \circ X=\mathrm{id}_{K}$ and such that for each $x_{0} \in K$, for each small open neighborhood $U$ of $x_{0}$, and for each coordinate system $u$ on $U$ with origin $x_{0}$, 


$$
X: U \longrightarrow T(U ; u) \subseteq U \times V(u)
$$

may be written in the form

$$
X(x)=\left(x, \sum_{i} f_{i}(x)\left(\partial / \partial u_{i}\right)_{x_{0}}\right)
$$

where each $f_{i}: U \rightarrow R$ is piecewise smooth.

Observe that each $f_{i}$ in Definition 2.1 is necessarily identically zero off St $u_{i}$ because of the definition of $T(U ; u)$. Thus if $X$ satisfies the condition of Definition 2.1 over the small open neighborhood $U$ of $x_{0}$ with respect to the coordinate system $u$, then $X$ automatically satisfies the condition over any small open neighborhood $W \subseteq U$ of $x_{1} \in U$ with respect to the coordinate system on $W$ with origin $x_{1}$ induced by $u$. Furthermore if $X$ satisfies the condition of Definition 2.1 with respect to the coordinate system $u$, then $X$ automatically satisfies the condition with respect to any other coordinate system on $U$ with origin $x_{0}$. Consequently, if $U$ is any small open subset of $K$, then a continuous vector field

$$
X: U \longrightarrow T U=\Perp T(U ; u) / \sim \text {. }
$$

Furthermore $X$ may be written with respect to a coordinate system $u$ on $U$ in the form $X=\sum_{i} f_{i}\left(\partial / \partial u_{i}\right)$, the value of $X$ at $x \in U$ being given by $X(x)=\sum_{i} f_{i}(x)\left(\partial / \partial u_{i}\right)_{x}$.

The set $\mathscr{X}(U)$ of continuous vector fields on a small open subset $U$ of a simplicial complex $K$ clearly forms a module over the ring $A(U)$ of piecewise smooth real-valued functions on $U$ with respect to pointwise operations. The set $\mathscr{P}(K)$ of globally defined continuous vector fields on $K$ similarly forms a module over the ring $A(K)$ of piecewise smooth real-valued functions on $K$.

We now focus on combinatorial manifolds. The reason for this is that we may use the following result.

Proposition 2.2. Let $M$ be a combinatorial n-manifold. Let $x_{0} \in M$, let $U$ be a small open neighborhood of $x_{0}$, and let $u=\left\{u_{1}, \cdots, u_{N}\right\}$ be a coordinate system on $U$ with origin $x_{0}$ for which $\left\{u_{1}, \cdots, u_{p}\right\}$ coordinatizes $U_{\alpha}=\sigma_{\alpha} \cap U$ where $x_{0}$ is contained in the interior of the p-simplex $\sigma_{\alpha} \subseteq M$. Then the support St $u_{i}$ of $u_{i}$ is $U$ if and only if $i=1, \cdots, p$.

(Note: The set $\left\{u_{1}, \cdots, u_{p}\right\}$ may be empty; this is the case, in fact, iff $x_{0}$ is a vertex. In the event that $x_{0}$ is a vertex, Proposition 2.2 states, in particular, that St $u_{i} \neq U$ for every $i$.)

Proof. The "if" part is clear; it is, in fact, true for simplicial complexes in general. 
For the "only if" part, using the standard metric on $R^{n}$ we write $R^{n}=R^{p} \oplus R^{n-p}$. Let $h: U \rightarrow V \subseteq R^{n}$ be a piecewise linear chart on $M$ for which the image of each wedge of $U$ is a wedge of $V$ and for which $h\left(U_{\alpha}\right) \leqq R^{p}$. Without loss of generality we may assume that for $i=p+1, \cdots, N, h_{*}\left(\partial / \partial u_{i}\right)_{x_{0}}$ is in $T_{h\left(x_{0}\right)} R^{n-p}$ and has unit length. (See Diagram 2.)

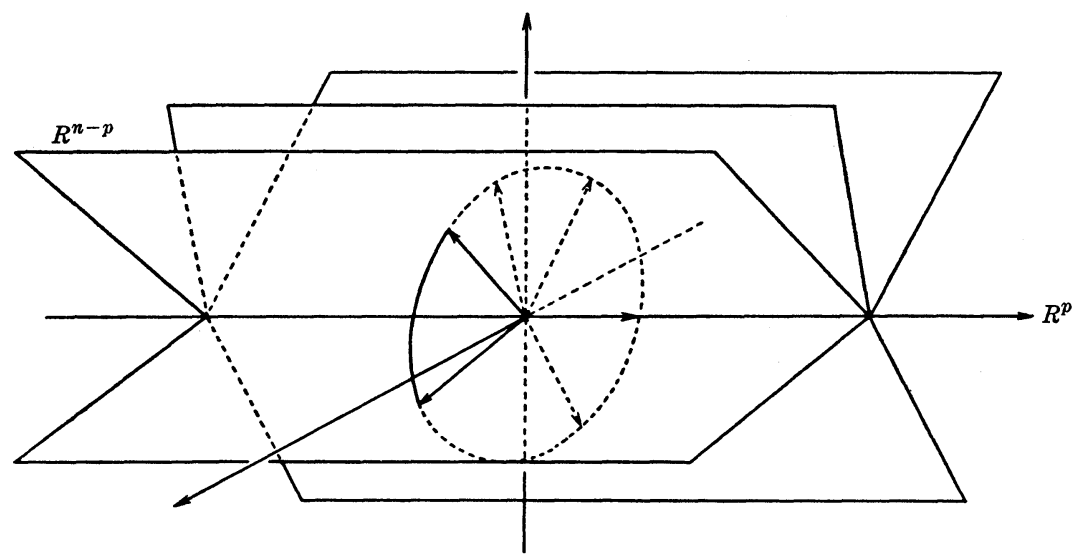

In this case $n=3, p=1$, and $N=6$.

DiAgram 2

If $S^{n-p-1}$ is the unit sphere in $R^{n-p}$, then the triangulation of $M$ determines a triangulation of $S^{n-p-1}$ with respect to which $S^{n-p-1}$ is a combinatorial manifold: The vertices $v_{i}$ of the triangulation are the endpoints of the vectors $h_{*}\left(\partial / \partial u_{i}\right)_{x_{0}}$, for $i=p+1, \cdots, N$, and $\left(v_{i_{1}}, \cdots, v_{i_{k}}\right)$ is a $k$-simplex of the triangulation iff

$$
\left\{u_{1}, \cdots, u_{p}, u_{i_{1}}, \cdots, u_{i_{k}}\right\}
$$

coordinatizes a $(p+k)$-wedge of $U$. To verify that this is a triangulation of $S^{n-p-1}$ one must verify that the intersection of any two simplices is a simplex, and that $S^{n-p-1}$ is covered by simplices. The first assertion is obvious. The second assertion follows since for any $y \in S^{n-p-1}$, the half line

$$
\left\{h\left(x_{0}\right)+t\left(y-h\left(x_{0}\right)\right) \mid t \in R^{+}\right\}
$$

must intersect $V$ in some point $y^{\prime}$ (since $V$ is an open neighborhood of $\left.h\left(x_{0}\right)\right)$; if $y^{\prime} \in h\left(U_{\beta}\right)$ for $U_{\beta}$ a wedge of $U$, and $U_{\beta}$ is coordinatized by

$$
\left\{u_{1}, \cdots, u_{p}, u_{i_{1}}, \cdots, u_{i_{k}}\right\}
$$

then $y \in\left(v_{i_{1}}, \cdots, v_{i_{k}}\right)$.

Now if $i=p+1, \cdots, N$ and St $u_{i}=U$, then the closed star of $v_{i}$ is $S^{n-p-1}$. But this is impossible since the closed star of every 
vertex of (the combinatorial manifold) $S^{n-p-1}$ has boundary, and $S^{n-p-1}$ does not.

The main results of this paper are stated for combinatorial manifolds. There are simplicial complexes other than combinatorial manifolds for which many of the following results are still valid, however: One could just as well work with any simplicial complex for which the conclusion of Proposition 2.2 holds. An example of such a simplicial complex (which is not a combinatorial manifold) is pictured in Diagram 3: There are five vertices and six 1-simplices.

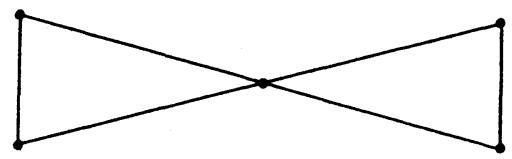

DIAGRAM 3

Recall (see Introduction) that all combinatorial manifolds in this paper are assumed to have no boundary. Observe (see Diagram 4) that Proposition 2.2 is no longer valid if one considers combinatorial manifolds with boundary: In this case $U$ is the open star of the vertex $x_{0}$ in $K$, there are no coordinate functions which coordinatize $U_{\alpha}=\left\{x_{0}\right\}$, and the support St $u_{1}$ of the coordinate function $u_{1}$ is $U$ (see the Note following the statement of Proposition 2.2). Even so, many later results can still be proved for combinatorial manifolds with boundary (and in fact for simplicial complexes in general) by using appropriate modifications of techniques presented here.

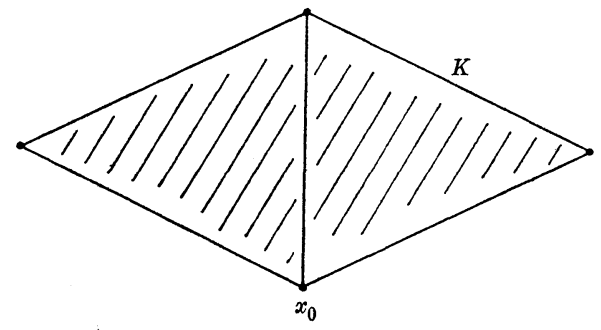

(a)

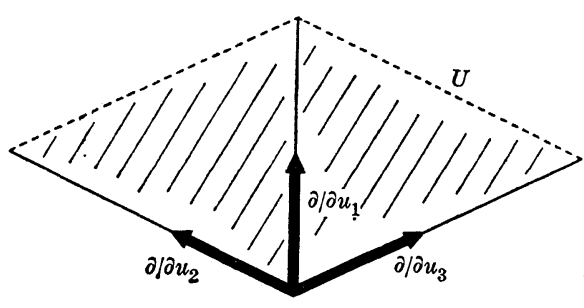

(b)

Diagram 4

The following result characterizes continuous vector fields on combinatorial manifolds locally.

Proposition 2.3. Let $M$ be a combinatorial manifold. Let $U \subseteq M$ be a small convex open neighborhood of $x_{0}$, and let $u=\left\{u_{1}, \cdots, u_{N}\right\}$ be a coordinate system on $U$ with origin $x_{0}$ for which $\left\{u_{1}, \cdots, u_{p}\right\}$ coordinatizes $U_{\alpha}=\sigma_{\alpha} \cap U$ where $x_{0}$ is contained in the interior of 
the $p$-simplex $\sigma_{\alpha} \subseteq M$. Then $X: U \rightarrow T U$ is a continuous vector field iff $X$ is of the form

$$
X=\sum_{i=1}^{p} f_{\imath}\left(\partial / \partial u_{i}\right)+\sum_{i=p+1}^{N} u_{i} g_{i}\left(\partial / \partial u_{i}\right)
$$

where $f_{i} \in A\left(\right.$ St $\left.u_{i}\right)=A(U)$ for $i=1, \cdots, p$, and $g_{i} \in A\left(\right.$ St $\left.u_{i}\right)$ for $i=$ $p+1, \cdots, N$, and St $u_{i}$ again denotes the support of $u_{i}$.

Note. In this proposition, and frequently throughout the sequel, for $g \in A\left(\operatorname{St} u_{i}\right), i=p+1, \cdots, N$, we consider $u_{i} g$ as an element of $A(U)$ by $\left(u_{i} g\right) x=u_{i}(x) g(x)$ if $x \in \mathrm{St} u_{i}$, and $\left(u_{i} g\right) x=0$ if $x \notin \mathrm{St} u_{i}$.

Before proving Proposition 2.3, let us first recall (see [6]) that one can think of elements $f$ of the ring $A(U)$ of piecewise smooth real-valued functions on $U$ as compatible tuples $\left(f_{\alpha}\right) \in \mathrm{X}_{\alpha} A\left(U_{\alpha}\right)$ of smooth real-valued functions $f_{\alpha} \in A\left(U_{\alpha}\right)$ defined on the wedges $U_{\alpha} \subseteq U$; here "compatible" means that if $U_{\alpha}$ and $U_{\beta}$ are wedges of $U$ then $\left.f_{\alpha}\right|_{U_{\alpha} \cap U_{\beta}}=\left.f_{\beta}\right|_{U_{\alpha} \cap U_{\beta}}$. If functions are expressed in this manner then for $f=\left(f_{\alpha}\right)$ and $g=\left(g_{\alpha}\right)$ in $A(U), f+g=\left(f_{\alpha}+g_{\alpha}\right)$ and $f \cdot g=\left(f_{\alpha} \cdot g_{\alpha}\right)$.

Also recall that for each $u_{i} \in u$ there is a derivation $\partial / \partial u_{i}: A(U) \rightarrow$ $A\left(\mathrm{St} u_{i}\right)$ defined by a compatible collection of derivations

$$
\left\{\partial / \partial u_{\alpha}^{i}: A\left(U_{\alpha}\right) \longrightarrow A\left(U_{\alpha}\right)\right\}
$$

defined for the wedges $U_{\alpha} \subseteq U$ for which $\left.u_{i}\right|_{U_{\alpha}}=u_{\alpha}^{i}$ is a coordinate function on $U_{\alpha}$; here "compatible" means that if $U_{\alpha}$ and $U_{\beta}$ are wedges of $U$ for which $\partial / \partial u_{\alpha}^{i}$ and $\partial / \partial u_{\beta}^{i}$ are defined, then

$$
\left.f_{\alpha}\right|_{U_{\alpha} \cap U_{\beta}}=\left.\left.f_{\beta}\right|_{U_{\alpha} \cap U_{\beta}} \Longrightarrow \frac{\partial f_{\alpha}}{\partial u_{\alpha}^{i}}\right|_{U_{\alpha} \cap U_{\beta}}=\left.\frac{\partial f_{\beta}}{\partial u_{\beta}^{i}}\right|_{U_{\alpha} \cap U_{\beta}} \cdot
$$

Observe that if $i=1, \cdots, p$ then St $u_{i}=U$ so that $\partial / \partial u_{i}: A(U) \rightarrow A(U)$.

Proof (of Proposition 2.3). If $X=\sum_{i} f_{i}\left(\partial / \partial u_{i}\right)$, then $f_{i}: U \rightarrow R$ is identically zero off St $u_{i}$ for each $u_{i} \in u$. Thus Proposition 2.2 implies that for $i=p+1, \cdots, N, f_{i}(x)=0$ if $u_{i}(x)=0$. Now by working wedgewise and then checking for compatibility, we find that the following calculation makes sense: If we consider $f_{i}$ as a function of one variable, namely $u_{i}$ (i.e., holding $u_{1}, \cdots, \widehat{u}_{i}, \cdots, u_{N}$ fixed), we have

$$
D_{i} f_{i}\left(u_{1}, \cdots, u_{N}\right)=D_{i} \int_{t=0}^{t=u_{i}} D_{i} f_{i}\left(u_{1}, \cdots, t, \cdots, u_{N}\right) d t,
$$

where $D_{i}$ is differentiation with respect to the $i$ th variable, so that

$$
f_{i}\left(u_{1}, \cdots, u_{N}\right)+C=\int_{t=0}^{t=u_{i}} D_{i} f_{i}\left(u_{1}, \cdots, t, \cdots, u_{N}\right) d t
$$


on St $u_{i}$. Evaluating at $u_{i}=0$ we find that $C=0$ so that after a change of variable (namely $t=u_{i} s$ ),

$$
f_{i}\left(u_{1}, \cdots, u_{N}\right)=u_{i} \int_{s=0}^{s=1} D_{i} f_{i}\left(u_{1}, \cdots, s u_{i}, \cdots, u_{N}\right) d s .
$$

Before stating the following corollary of Proposition 2.3, let us first recall (see [6]) that for $i=p+1, \cdots, N$, any $f \in A\left(\right.$ St $\left.u_{i}\right)$ can be extended to an $\bar{f} \in A(U)$; such extensions are not unique.

CoRollary 2.4. The $A(U)$-module $\mathscr{X}(U)$ is finitely generated by $\partial / \partial u_{i}$ for $i=1, \cdots, p$, and $u_{i}\left(\partial / \partial u_{i}\right)$ for $i=p+1, \cdots, N$. In fact for $f$ in $A(U)$,

$$
X=\sum_{i=1}^{p} f_{i}\left(\partial / \partial u_{i}\right)+\sum_{i=p+1}^{N} u_{i} g_{i}\left(\partial / \partial u_{i}\right)
$$

and

$$
Y=\sum_{i=1}^{p} h_{i}\left(\partial / \partial u_{i}\right)+\sum_{i=p+1}^{N} u_{i} k_{i}\left(\partial / \partial u_{i}\right)
$$

in $\mathscr{Z}(U)$ we have

$$
f \cdot X=\sum_{i=1}^{p} f f_{i}\left(\partial / \partial u_{i}\right)+\sum_{i=p+1}^{N} u_{i}\left(\left.f\right|_{\mathrm{st} u_{i}}\right) g_{i}\left(\partial / \partial u_{i}\right),
$$

and

$$
X+Y=\sum_{i=1}^{p}\left(f_{i}+h_{i}\right)\left(\partial / \partial u_{i}\right)+\sum_{i=p+1}^{N} u_{i}\left(g_{i}+k_{i}\right)\left(\partial / \partial u_{i}\right) .
$$

Observe that $\mathscr{Q}(U)$ is not, in general, free over $A(U)$ since for $i=p+1, \cdots, N, f^{\prime}, f^{\prime \prime} \in A(U)$ may agree on St $u_{i}$, so that

$$
\begin{aligned}
f^{\prime} \cdot\left(u_{i}\left(\partial / \partial u_{i}\right)\right) & =u_{i}\left(\left.f^{\prime}\right|_{\mathrm{St} u_{i}}\right)\left(\partial / \partial u_{i}\right) \\
& =u_{i}\left(\left.f^{\prime \prime}\right|_{\mathrm{st} u_{i}}\right)\left(\partial / \partial u_{i}\right)=f^{\prime \prime} \cdot\left(u_{i}\left(\partial / \partial u_{i}\right)\right),
\end{aligned}
$$

although $f^{\prime}$ and $f^{\prime \prime}$ clearly need not agree on all of $U$.

Continuous vector fields on combinatorial manifolds "lie along simplices" in the following sense.

Corollary 2.5. Let $M$ be a combinatorial manifold. If $X €$ $\mathscr{X}(M)$, then

(1) for every simplex $\sigma_{\alpha} \subseteq M$ there is a smooth vector field $X_{\alpha}: \sigma_{\alpha} \rightarrow T \sigma_{\alpha}$ defined on $\sigma_{\alpha}$ for which the following diagram commutes 


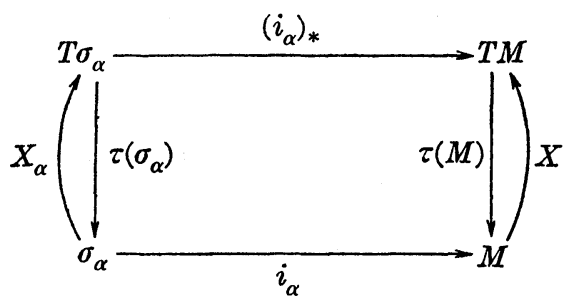

where $i_{\alpha}$ denotes inclusion and $\left(i_{\alpha}\right)_{*}$ denotes the Jacobian of $i_{\alpha}$, and

(2) for simplices $\sigma_{\alpha}, \sigma_{\beta} \subseteq M$ for which $\sigma_{\alpha} \subseteq \sigma_{\beta}$, the following diagram commutes naturally

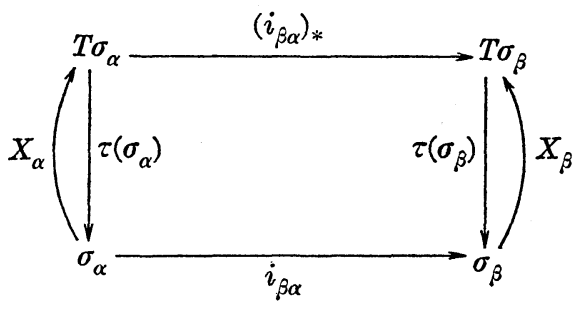

where $i_{\beta \alpha}$ denotes inclusion and $\left(i_{\beta_{\alpha}}\right)^{*}$ denotes the Jacobian of $i_{\beta_{\alpha}}$.

Conversely, given any collection $\left\{X_{\alpha}: \sigma_{\alpha} \rightarrow T \sigma_{\alpha}\right\}$ of vector fields defined on the simplices $\sigma_{\alpha} \subseteq M$ which satisfies Condition 2 above, there is a unique continuous vector field $X: M \rightarrow T M$ on $M$ which induces $X_{\alpha}: \sigma_{\alpha} \rightarrow T \sigma_{\alpha}$ on each simplex $\sigma_{\alpha} \subseteq M$ and for which Condition 1 holds.

In particular, a continuous vector field on a combinatorial manifold $M$ has a zero at every vertex of $M$.

To state the next corollary, let us first recall (see [7]) that a piecewise smooth flow $F$ on a simplicial complex $K$ is a piecewise smooth right action $F: K \times R \rightarrow K$ of the additive group of reals on $K$. Also recall that there is a distinguished type of vector field on a simplicial complex, namely the integrable vector fields, and there is a correspondence between integrable vector fields on $K$ and piecewise smooth flows on $K$.

Corollary 2.6. Let $M$ be a combinatorial manifold. Then every continuous vector field on $M$ is integrable. Furthermore there is a bijection between continuous vector fields on $M$ and piecewise smooth flows $F: M \times R \rightarrow M$ for which $F: \sigma_{\alpha} \times R \rightarrow \sigma_{\alpha}$ for each simplex $\sigma_{\alpha} \subseteq M$.

Proof. This is an immediate consequence of Corollary 2.5 and the following lemma. 
LEMMA 2.7. Let $U$ and $W$ be open neighborhoods of

$$
0 \in s^{n . p}=\left\{x=\left(x_{i}\right) \in R^{n} \mid x_{i} \geqq 0 \text { for } \quad i=p+1, \cdots, N\right\}
$$

for which the closure of $W$ is contained in $U$. Then for every smooth vector field

$$
X=\sum_{i=1}^{p} f_{i}\left(\partial / \partial x_{i}\right)+\sum_{i=p+1}^{N} x_{i} g_{i}\left(\partial / \partial x_{i}\right)
$$

on $U$ there is a unique smooth flow $F=\left(F_{i}\right): W \times I \rightarrow U$ for which

$$
\left.\frac{\partial F_{i}}{\partial t}\right|_{t=0}= \begin{cases}f_{i} & \text { for } i=1, \cdots, p \\ x_{i} g_{i} & \text { for } i=p+1, \cdots, N\end{cases}
$$

and $F:\left(s_{\alpha} \cap W\right) \times I \rightarrow s_{\alpha} \cap U$ for each face $s_{\alpha}$ of $s^{n, p}$.

This completes the proof of Corollary 2.6.

3. The main results. We will now prove the main results of this paper.

THEOREM 3.1. Let $M$ be a combinatorial manifold. There is an $A(M)$-module isomorphism between the module $\mathscr{X}(M)$ of continuous vector fields on $M$ and the module $\mathscr{D}(M)$ of derivations of $A(M)$.

Proof. It suffices to prove this result locally, so let $U$ be a small convex open neighborhood of $x_{0} \in M$, and let $u=\left\{u_{1}, \cdots, u_{N}\right\}$ be a coordinate system on $U$ for which $\left\{u_{1}, \cdots, u_{p}\right\}$ coordinatizes $U_{\alpha}=\sigma_{\alpha} \cap U$ where $x_{0}$ is contained in the interior of the $p$-simplex $\sigma_{\alpha} \subseteq M$. We define

$$
F: \mathscr{Z}(U) \longrightarrow \mathscr{D}(U)
$$

by associating to the vector field (see Proposition 2.3)

$$
X=\sum_{i=1}^{p} f_{i}\left(\partial / \partial u_{i}\right)+\sum_{i=p+1}^{N} u_{i} g_{i}\left(\partial / \partial u_{i}\right)
$$

the derivation

$$
F(X): f \longmapsto \sum_{i=1}^{p} f_{i}\left(\partial f / \partial u_{i}\right)+\sum_{i=p+1}^{N} u_{i} g_{i}\left(\partial f / \partial u_{i}\right) .
$$

This makes sense: Since $f_{i}$ and $\partial f / \partial u_{i}$ are in $A(U)$ for $i=1, \cdots, p$, $f_{i}\left(\partial f / \partial u_{i}\right) \in A(U)$. Since $g_{i}$ and $\partial f / \partial u_{i}$ are in $A\left(\right.$ St $\left.u_{i}\right)$ for $i=p+1$, $\cdots, N, g_{i}\left(\partial f / \partial u_{i}\right) \in A\left(\right.$ St $\left.u_{i}\right)$; thus $u_{i} g_{i}\left(\partial f / \partial u_{i}\right) \in A(U)$.

Clearly $F: \mathscr{Z}(U) \rightarrow \mathscr{D}(U)$ is an $A(U)$-module homomorphism. Moreover it is injective: If $X \in \mathscr{Z}(U)$ is a continuous vector field 
as above and $F(X)=0$, then $(F(X)) u_{i}=0$ for every $i$. If $i=1$, $\cdots, p$, this means that $f_{i}=0$ on $U$, and if $i=p+1, \cdots, N$, this means that $u_{i} g_{i}=0$ on $U$. Thus $X=0$.

Finally we show that $F: \mathscr{X}(U) \rightarrow \mathscr{D}(U)$ is surjective as follows: Given a derivation $D: A(U) \rightarrow A(U)$, let $f_{i}=D u_{i}$ for $i=1, \cdots, N$. Then since $D$ is a derivation it is easy to see that for $i=p+1$, $\cdots, N, f_{i}(x)=0$ for $x \notin \mathrm{St} u_{i}$. Consequently $f_{i}=u_{i} g_{i}$, for $i=p+1$, $\cdots, N$, where $g_{i} \in A\left(\mathrm{St} u_{i}\right)$ (see the proof of Proposition 2.3). The surjectivity of $F$ will follow from showing that

$$
D=\sum_{i=1}^{p} f_{i}\left(\partial / \partial u_{i}\right)+\sum_{i=p+1}^{N} u_{i} g_{i}\left(\partial / \partial u_{i}\right)
$$

so that $D=F(X)$ where

$$
X=\sum_{i=1}^{p} f_{i}\left(\partial / \partial u_{i}\right)+\sum_{i=p+1}^{N} u_{i} g_{i}\left(\partial / \partial u_{i}\right) .
$$

To show this we need the following lemma.

LemMA 3.2. Let $W$ be a small open neighborhood of $x_{1}$ which is star shaped with respect to $x_{1}$, and let $w$ be a coordinate system on $W$ with origin $x_{1}$. Then for every $f \in A(W)$ and $w_{i} \in w$ there is a piecewise smooth function $\varphi_{i} \in A\left(\mathrm{St} w_{i}\right)$ such that $\varphi_{i}\left(x_{1}\right)=\left(\partial f / \partial w_{i}\right) x_{1}$ and $f=f\left(x_{1}\right)+\sum_{i} w_{i} \varphi_{i}$ on $W$ (where $f\left(x_{1}\right)$ is a constant function on $W$ ).

This lemma is proved by first verifying a similar result on each wedge of $W$ (see (2) for the classical smooth analog), and then verifying that these similar results are compatible with respect to restriction.

Now to finish the proof of surjectivity (again compare with (2)), let $x_{1} \in U$, let $W \subseteq U$ be a small star shaped open neighborhood of $x_{1}$, and let

$$
w=\left\{u_{i}-u_{i}\left(x_{1}\right): W \rightarrow R \mid u_{i} \in u \text { and }\left.u_{i}\right|_{W} \neq 0\right\}
$$

be the coordinate system on $W$ with origin $x_{1}$ induced by $u$. Observe that for $i=1, \cdots, p, w_{i}=u_{i}-u_{i}\left(x_{1}\right)$ is in $w$.

We let $I$ denote the index set of $w$ for convenience.

Given any $f \in A(U)$ we apply Lemma 3.2 to $\left.f\right|_{W}$ and observe that for each $i \in I,\left(\partial f / \partial w_{i}\right) x_{1}=\left(\partial f / \partial u_{i}\right) x_{1}$ to obtain

$$
\begin{aligned}
(D f) x_{1} & =\left(D\left(\left.f\right|_{W}\right)\right) x_{1} \\
& =\left(D\left(f\left(x_{1}\right)+\sum_{i \in I} w_{i} \varphi_{i}\right)\right) x_{1} \\
& =\sum_{i \in I} D\left(w_{i} \bar{\varphi}_{i}\right) x_{1} \\
& =\sum_{i \in I}\left(\left(D w_{i}\right) \bar{\varphi}_{i}+w_{i}\left(D \bar{\varphi}_{i}\right)\right) x_{1}
\end{aligned}
$$


where $\bar{\varphi}_{i}=\varphi_{i}$ if St $w_{i}=W$, and where $\bar{\varphi}_{i} \in A(W)$ is an extension of $\varphi_{i} \in A\left(\mathrm{St} w_{i}\right)$ otherwise. Thus,

$$
\begin{aligned}
(D f) x_{1} & =\sum_{i \in I}\left(\left(D\left(u_{i}-u_{i}\left(x_{1}\right)\right)\right) \varphi_{i}\right) x_{1} \\
& =\sum_{i \in I}\left(\left(D u_{i}\right)\left(\partial f / \partial w_{i}\right)\right) x_{1} \\
& =\sum_{i \in I}\left(f_{i}\left(\partial f / \partial u_{1}\right)\right) x_{i} \\
& =\left(\sum_{i=1}^{p} f_{i}\left(\partial f / \partial u_{i}\right)+\sum_{i \in I-\{1, \ldots, p\}} f_{i}\left(\partial f / \partial u_{i}\right)\right) x_{1} \\
& =\left(\sum_{i=1}^{p} f_{i}\left(\partial f / \partial u_{i}\right)+\sum_{i \in I-\{1, \ldots, p\}} u_{i} g_{i}\left(\partial f / \partial u_{i}\right)\right) x_{1} \\
& =\left(\sum_{i=1}^{p} f_{i}\left(\partial f / \partial u_{i}\right)+\sum_{i=p+1}^{N} u_{i} g_{i}\left(\partial f / \partial u_{i}\right)\right) x_{1},
\end{aligned}
$$

the last equality following since $u_{i}\left(x_{1}\right)=0$ if $i \notin I$.

CoROLLARY 3.3. The $A(M)$-module $\mathscr{P}(M)$ of continuous vector fields on a combinatorial manifold $M$ is a Lie algebra over $R$ with respect to the bracket operation

$$
[X, Y] f=X(Y f)-Y(X f)
$$

for $X, Y \in \mathscr{Q}(M)$ and $f \in A(M)$.

In fact it is easy to show that if $F, G: M \times R \rightarrow M$ are integral flows for $X, Y \in \mathscr{Q}(M)$, then $[Y, X]$ is the derivative at $0 \in R^{+}$of the map from $M \times R^{+}$to $M$ given by

$$
(x, t) \longmapsto G(F(G(F(x, \sqrt{t}), \sqrt{t}),-\sqrt{t}),-\sqrt{t}) .
$$

This is completely analogous to the smooth case (see [8]).

The next corollary is an application of Theorem 3.1 to PL manifolds. By a closed $P L n$-manifold $\underline{M}$ we mean a closed topological $n$-manifold $M$ together with an equivalence class of triangulations $T: M \rightarrow \underline{M}$ of $\underline{M}$ by closed combinatorial $n$-manifolds $M$, two triangulations being equivalent iff they have a common subdivision; the equivalence class is the "PL structure" of $\underline{M}$.

If $\underline{M}$ is a closed PL $n$-manifold, we let $A(\underline{M})$ denote the ring of continuous functions $f: \underline{M} \rightarrow R$ such that for some triangulation $T: M \rightarrow \underline{M}$ in the PL structure of $\underline{M}, f \circ T \in A(M)$.

CoROLlary 3.4. Let $\underline{M}$ be a closed $P L$ manifold, and let $\underline{D}: A(\underline{M}) \rightarrow A) \underline{M})$ be a derivation which satisfies the following property: for every triangulation $T: M \rightarrow \underline{M}$ of $\underline{M}$ by a combinatorial manifold $M$ in the $P L$ structure of $\underline{M}$ there is a derivation $D: A(M) \rightarrow A(M)$ for which the following diagram commutes 


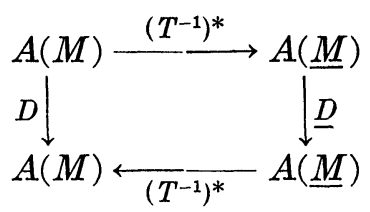

where $\left(T^{-1}\right)^{*} f=f \circ T^{-1}: \underline{M} \rightarrow R$ for $f \in A(M)$. Then $\underline{D}=0$.

Proof. Let $\underline{f} \in A(\underline{M})$ and $x_{0} \in \underline{M}$. We will show that $(\underline{D f}) x_{0}=0$ : Let $T: M \rightarrow \underline{M}$ be a triangulation in the PL structure of $\underline{M}$ for which $f \circ T \in A(M)$ and for which $T^{-1}\left(x_{0}\right)$ is a vertex of $M$. Then the associated derivation $D$ of $A(M)$ corresponds to a continuous vector field on $M$ which is necessarily zero at $x_{0}$. Thus

$$
(\underline{D f}) x_{0}=\underline{D}\left(T^{-1}\right)^{*}(f \circ T) x_{0}=\left(T^{-1}\right)^{*} D(f \circ T) x_{0}=(D(f \circ T))\left(T^{-1}\left(x_{0}\right)\right)=0 .
$$

Now let $K$ be a simplicial complex and let $U$ be a small open subset of $K$. Recall (see [6]) that the $A(U)$-module $E(U)$ of piecewise smooth 1-forms on $U$ consists of all compatible tuples $\left(\theta_{\alpha}\right) \epsilon$ $\mathrm{X}_{\alpha} E\left(U_{\alpha}\right)$ of smooth 1 -forms $\theta_{\alpha} \in E\left(U_{\alpha}\right)$ defined on the wedges $U_{\alpha} \subseteq U$; here "compatible" means that if $U_{\alpha}$ and $U_{\beta}$ are wedges of $U$ then $\left.\theta_{\alpha}\right|_{U_{\alpha} \cap U_{\beta}}=\left.\theta_{\beta}\right|_{U_{\alpha} \cap U_{\beta}}$. If 1-forms are expressed in this manner then for $f=\left(f_{\alpha}\right)$ in $A(U), \theta=\left(\theta_{\alpha}\right)$ and $\phi=\left(\varphi_{\alpha}\right)$ in $E(U), f \cdot \theta=\left(f_{\alpha} \cdot \theta_{\alpha}\right)$ and $\theta+\varphi=\left(\theta_{\alpha}+\varphi_{\alpha}\right)$; i.e., operations are wedgewise.

Alternately, if $u$ is a coordinate system on $U$ then $\theta \in E(U)$ may be expressed $\theta=\sum_{i} f_{i} d u_{i}$ where each $f_{i} \in A\left(\right.$ St $\left.u_{i}\right)$, St $u_{i}$ denoting the support of $u_{i}$. If forms are expressed in this manner then for $f$ in $A(U), \theta=\sum_{i} f_{i} d u_{i}$ and $\varphi=\sum_{i} g_{i} d u_{i}$ in $E(U), f \cdot \theta=\sum_{i}\left(\left.f\right|_{\mathrm{St}_{i}}\right) f_{i} d u_{i}$ and $\theta+\varphi=\sum_{i}\left(f_{i}+g_{i}\right) d u_{i}$.

THEOREM 3.5. Let $M$ be a combinatorial manifold. There is an $A(M)$ module isomorphism between the module $E(M)$ of global piecewise smooth 1-forms on $M$ and the module $\mathrm{Hom}_{A(M)}(\mathscr{X}(M), A(M))$ of $A(M)$ linear maps from $\mathscr{P}(M)$ to $A(M)$.

Proof. It again suffices to prove this result locally, so, with the notation of Theorem 3.1, we define

$$
F: E(U) \longrightarrow \operatorname{Hom}_{A(U)}(\mathscr{X}(U), A(U))
$$

by associating to the 1 -form $\theta=\sum_{i} f_{i} d u_{i}$ the homomorphism

$$
F(\theta): \sum_{i=1}^{p} g_{i}\left(\partial / \partial u_{i}\right)+\sum_{i=p+1}^{N} u_{i} h_{i}\left(\partial \partial u_{i}\right) \longmapsto \sum_{i=1}^{p} f_{i} g_{i}+\sum_{i=p+1}^{N} u_{i} f_{i} h_{i} \text {. }
$$

This makes sense: Since $f_{i}$ and $g_{i}$ are in $A(U)$ for $i=1, \cdots, p$, clearly $f_{i} g_{i} \in A(U)$. Since $f_{i}$ and $h_{i}$ are in $A\left(\right.$ St $\left.u_{i}\right)$ for $i=p+1, \cdots$, $N$, we have $f_{i} h_{i} \in A\left(\right.$ St $\left.u_{i}\right)$; thus $u_{i} f_{i} h_{i} \in A(U)$. 
Clearly $F: E(U) \rightarrow \operatorname{Hom}_{A(U)}(\mathscr{Q}(U), A(U))$ is an $A(U)$-module homomorphism. Moreover it is injective: If $\theta=\sum_{i} f_{i} d u_{i}$ is in $E(U)$ and $F(\theta)=0$, then for $i=1, \cdots, p, f_{i}=(F(\theta)) d u_{i}=0$ on $U$, and for $i=$ $p+1, \cdots, N, u_{i} f_{i}=(F(\theta)) u_{i}\left(\partial / \partial u_{i}\right)=0$ on $U$; thus $\theta=0$.

Finally we show that $F: E(U) \rightarrow \operatorname{Hom}_{A(U)}(\mathscr{X}(U), A(U))$ is surjective as follows: Given $f \in \operatorname{Hom}_{A(U)}(\mathscr{Z}(U), A(U))$, let $f_{i}=f\left(\partial / \partial u_{i}\right)$ for $i=$ $1, \cdots, p$ and $f_{i}=f\left(u_{i}\left(\partial / \partial u_{i}\right)\right)$ for $i=p+1, \cdots, N$. Since $f$ is $A(U)$ linear, it is easy to see that $f_{i}=f\left(u_{i}\left(\partial / \partial u_{i}\right)\right)=0$ off St $u_{i}$ for $i=$ $p+1, \cdots, N$. Thus, as before (see the proof of Proposition 2.3), $f_{i}=u_{i} g_{i}$, for $i=p+1, \cdots, N$, where $g_{i} \in A\left(\right.$ St $\left.u_{i}\right)$. Consequently if

$$
\theta=\sum_{i=1}^{p} f_{i} d u_{i}+\sum_{i=p+1}^{\mathrm{N}} g_{i} d u_{i},
$$

then clearly $F(\theta)=f$.

In order to generalize the previous result, recall (see [6]) that if $K$ is a simplicial complex and $U$ is a small open subset of $K$ then the $A(U)$-module $\Lambda^{q} E(U)$ of piecewise smooth $q$-forms on $U$ consists of all compatible tuples $\left(\theta_{\alpha}\right) \in \mathrm{X}_{\alpha} \Lambda^{q} E\left(U_{\alpha}\right)$ of smooth $q$-forms $\theta_{\alpha} \in \Lambda^{q} E\left(U_{\alpha}\right)$ defined on the wedges $U_{\alpha} \subseteq U$; here "compatibility" is defined as before (i.e., as for $E(U)$ ), and the module operations are again wedgewise.

Alternately, if $u$ is a coordinate system on $U$ then $\theta \in \Lambda^{q} E(U)$ may be expressed $\theta=\sum_{i} f_{i} d u_{i_{1}} \cdots d u_{i_{q}}$ where the summation is taken over all $i=\left(i_{1}, \cdots, i_{q}\right)$ for which there is a wedge $U_{\alpha} \subseteq U$ such that $\left.u_{i_{1}}\right|_{U_{\alpha}}, \cdots,\left.u_{i_{q}}\right|_{U_{\alpha}}$ are all coordinate functions on $U_{\alpha}$ and where $f_{i} \in$ $A\left(\bigcap_{j=1}^{q} \mathrm{St} u_{i_{j}}\right)$; for convenience we will use multi-index notation to write $\theta=\sum_{i} f_{i} d u_{i}$ where $f_{i} \in A\left(\mathrm{St} u_{i}\right)$. If $q$-forms are expressed in this manner, then for $f$ in $A(U), \theta=\sum_{i} f_{i} d u_{i}$ and $\varphi=\sum_{i} g_{i} d u_{i}$ in $\Lambda^{q} E(U), f \cdot \theta=\sum_{i}\left(\left.f\right|_{\mathrm{stu}_{i}}\right) f_{i} d u_{i}$ ond $\theta+\varphi=\sum_{i}\left(f_{i}+g_{i}\right) d u_{i}$.

Observe that since the index of summation for $\theta=\sum_{i} f_{i} d u_{i}$ in $\Lambda^{q} E(U)$ is restricted, $\Lambda^{q} E(U)$ is not the $q$-fold exterior product of $E(U)$ : Actually $\Lambda^{q} E(U)$ is the q-fold exterior product $E(U)$ with the added relation that $d u_{i_{1}} \cdots d u_{i_{q}}=0$ if there is no wedge $U_{\alpha} \leqq U$ for which $\left.u_{i_{1}}\right|_{U_{\alpha}}, \cdots,\left.u_{i_{q}}\right|_{U_{\alpha}}$ are all coordinate functions on $U_{\alpha}$. We will continue to use the notation $\Lambda^{q} E(U)$ for the module of piecewise smooth $q$-forms on $U$ since the $q$-fold exterior product of $E(U)$ will not be used in the sequel.

THEOREM 3.6. Let $M$ be a combinatorial manifold. There is an $A(M)$-module isomorphism between the module $\Lambda^{q} E(M)$ of global piecewise smooth $q$-forms on $M$ and the module $\mathrm{Alt}_{A(M)}\left(\mathrm{X}^{q} \mathscr{X}(M), A(M)\right)$ of $A(M)$-linear alternating maps from the $q$-fold product $\times^{q} \mathscr{X}(M)$ of $\mathscr{X}(M)$ to $A(M)$. 
Proof. Once again it suffices to prove the result locally, so again with the notation of Theorem 3.1 we define

$$
F: \Lambda^{q} E(U) \longrightarrow \operatorname{Alt}_{A(U)}\left(X^{q} \mathscr{Q}(U), A(U)\right)
$$

by defining it on forms $f_{i} d u_{i}$ and extending to $\Lambda^{q} E(U)$ by $A(U)$ linearity; the map $F\left(f_{i} d u_{i_{1}} \cdots d u_{i_{q}}\right)$ takes $\left(g_{j_{1}}\left(\partial / \partial u_{j_{1}}\right), \cdots, g_{j_{q}}\left(\partial / \partial u_{j_{q}}\right)\right)$ to $(-1)^{\pi} f_{i} g_{j_{1}} \cdots g_{j_{q}}$ if there is a permutation $\pi$ taking $i=\left(i_{1}, \cdots, i_{q}\right)$ to $\left(j_{1}, \cdots, j_{q}\right)$, and 0 otherwise. (Note that for $j=p+1, \cdots, N$, we write $u_{j} h_{j}\left(\partial / \partial u_{j}\right)=g_{j}\left(\partial / \partial u_{j}\right)$.) As before it is easy to verify that $F$ is well defined and an isomorphism.

Finally recall (see [6]) that if $K$ is a simplicial complex, $U$ is a small open subset of $K$, and $u$ is a coordinate system on $U$, then the differential $d$ of the de Rham complex $\left(\Lambda^{*} E(U), d\right)$ of $U$ is defined wedgewise but may alternately be described by

$$
d \theta=d\left(\sum_{i} f_{i} d u_{i_{1}} \cdots d u_{i_{q}}\right)=\sum_{i_{0}, i}\left(\partial f_{i} / \partial u_{i_{0}}\right) d u_{i_{0}} d u_{i_{1}} \cdots d u_{i_{q}},
$$

the summation taken over all $i_{0}, i$ for which $\left(\partial f_{i} / \partial u_{i_{0}}\right) d u_{i_{0}} d u_{i_{1}} \cdots d u_{i_{q}}$ is a $(q+1)$-form on $U$.

THEOREM 3.7. Let $M$ be a combinatorial manifold. If $d: \Lambda^{*} E(M) \rightarrow$ $\Lambda^{*} E(M)$ is the differential of the de Rham complex of $M$ and $\theta \in \Lambda^{q} E(M)$, then $d \theta \in \Lambda^{q+1} E(M)$ is given by the formula

$$
\begin{aligned}
d \theta\left(X_{1}, \cdots, X_{q+1}\right)= & \sum_{j}(-1)^{j+1} X_{j} \theta\left(X_{1}, \cdots, \hat{X}_{j}, \cdots, X_{q+1}\right) \\
& +\sum_{i<j}(-1)^{i+j} \theta\left(\left[X_{i}, X_{j}\right], X_{1}, \cdots, \hat{X}_{i}, \cdots, \hat{X}_{j}, \cdots, \hat{X}_{q+1}\right)
\end{aligned}
$$

for $X_{1}, \cdots, X_{q+1} \in \mathscr{P}(M)$.

Proof. Working locally with the notation of Theorem 3.1 and using the fact that $d, \theta$, and $X_{1}, \cdots, X_{q+1}$ are all additive, one need only prove the result in the case $\theta=f d u_{i_{1}}, \cdots, d u_{i_{q}}$, and $X_{1}=g_{j_{1}}\left(\partial / \partial u_{j_{1}}\right), \cdots$, $X_{q}=g_{j_{q}}\left(\partial / \partial u_{j_{q}}\right)$ (where for $j=p+1, \cdots, N$ we again write $u_{j} h_{j}\left(\partial / \partial u_{j}\right)=$ $\left.g_{j}\left(\partial / \partial u_{j}\right)\right)$. The result in this case is a straightforward calculation.

4 Connexions on combinatorial manifolds. Connexions exist (in tangent bundles) on smooth manifolds, and can be described in various equivalent ways; also such connexions can be interpreted geometrically in terms of parallel translation. The goal of this section is to establish analogous results for combinatorial manifolds. In particular we will establish the existence of connexions on combinatorial manifolds, and show that such connexions can be described (or defined) equivalently in terms of differential forms (Cartan connexions), vector 
fields (Koszul connexions) or compatible collections of connexions defined on individual simplices. Finally, such connexions are interpreted geometrically in terms of parallel translation.

Throughout this section, $M$ will be a combinatorial $n$-manifold (without boundary).

Definition 4.1. For every small open subset $U \subseteq M$, let $E(U) \otimes$ $E(U)$ denote the set of all tuples

$$
\omega=\left(\omega_{\alpha}\right)=\left(\sum_{i} \theta_{\alpha}^{i} \otimes \varphi_{\alpha}^{i}\right) \in \mathbf{X}_{\alpha}\left(E\left(U_{\alpha}\right){\underset{A(U)}{\alpha})}_{B} E\left(U_{\alpha}\right)\right),
$$

the product taken over all wedges $U_{\alpha} \leqq U$, for which $\left.\omega_{\alpha}\right|_{U_{\alpha} \cap U_{\beta}}=$ $\left.\omega_{\beta}\right|_{U_{\alpha} \cap U_{\beta}}$ for wedges $U_{\alpha}, U_{\beta} \cong U$, where

$$
\left.\omega_{\alpha}\right|_{U_{\alpha} \cap U_{\beta}}=\left.\left.\sum_{i} \theta_{\alpha}^{i}\right|_{U_{\alpha} \cap U_{\beta}} \otimes \varphi_{\alpha}^{i}\right|_{U_{\alpha} \cap U_{\beta}} \in E\left(U_{\alpha} \cap U_{\beta}\right) \underset{\left.A_{(U,} \cap U_{\alpha}\right)}{\bigotimes} E\left(U_{\alpha} \cap U_{\beta}\right) .
$$

Clearly $E(U) \otimes E(U)$ is an $A(U)$-module with respect to wedgewise operations. The construction of $E(U) \otimes E(U)$ is natural with respect to restriction to small open subsets $W \subseteq U$, and hence defines a presheaf on $M$. The associated sheaf is fine since piecewise smooth partitions of unity subordinate to covers of $M$ by small open subsets exist on $M$ (see [6]).

Definition 4.2. The $A(M)$-module $E(M) \otimes E(M)$ is the module of global sections of the sheaf over $M$ associated to the presheaf which assigns to each small open subset $U \subseteq M$ the $A(U)$-module $E(U) \otimes E(U)$.

As in the case of piecewise smooth forms, if $u$ is a coordinate system on the small open subset $U \subseteq M$, then each $\omega \in E(U) \otimes E(U)$ may be written in the form $\omega=\sum_{i, j} f_{i j} d u_{i} \otimes d u_{j}$ where the summation is taken over all $i$ and $j$ for which there is a wedge $U_{\alpha} \subseteq U$ such that $u_{i \mid U_{\alpha}}$ and $u_{j \mid U_{\alpha}}$ are coordinate functions on $U_{\alpha}$, and where $f_{i j} \in A\left(\mathrm{St} u_{i} \cap \mathrm{St} u_{j}\right)$, St $u_{i} \cap \mathrm{St} u_{j}$ the intersection of the supports of $u_{i}$ and $u_{j}$. Furthermore for $f$ in $A(U), \psi=\sum_{i, j} f_{i j} d u_{i} \otimes d u_{j}$ and $\omega=\sum_{i, j} g_{i j} d u_{i} \otimes d u_{j}$ in $E(U) \otimes E(U)$,

$$
f \cdot \psi=\sum_{i, j}\left(\left.f\right|_{\mathrm{stu} i_{i} \cap \mathrm{st} u_{j}}\right) f_{i j} d u_{i} \otimes d u_{j}
$$

and

$$
\psi+\omega=\sum_{i, j}\left(f_{i j}+g_{i j}\right) d u_{i} \otimes d u_{j}
$$

where the summations are restricted as above.

Since the index of summation for elements $\omega=\sum_{i, j} f_{i j} d u_{i} \otimes d u_{j}$ of $E(U) \otimes E(U)$ is restricted, $E(U) \otimes E(U)$ is not the tensor product 
of $E(U)$ with itself; actually $E(U) \otimes E(U)$ is the tensor product of $E(U)$ with itself with the added relation that $d u_{i} \otimes d u_{j}=0$ if there is no wedge $U_{\alpha} \leqq U$ for which $u_{i \mid U_{\alpha}}$ and $u_{j \mid U_{\alpha}}$ are both coordinate functions on $U_{\alpha}$. We will continue to use the notation $E(U) \otimes E(U)$ since the tensor product of $E(U)$ with itself will not be used in the sequel.

We now describe connexions on $M$ by differential forms.

Definition 4.3. A Cartan connexion on $M$ is a real linear map

$$
D: E(M) \longrightarrow E(M) \otimes E(M)
$$

for which $D(f \theta)=d f \otimes \theta+f D \theta$ for $f \in A(M)$ and $\theta \in E(M)$.

As in the smooth case, a Cartan connexion $D$ on $M$ uniquely determines a Cartan connexion

$$
D_{U}: E(U) \longrightarrow E(U) \otimes E(U)
$$

for every small open subset $U \subseteq M$.

Henceforth " $\mathscr{C}$ " denotes "set complement", and St $u_{i}$ again denotes the support of the coordinate function $u_{i}$.

THEOREM 4.4. Let $U \subseteq M$ be a small open subset, and let $u$ be a coordinate system on $U$. Then

$$
D: E(U) \longrightarrow E(U) \otimes E(U)
$$

is a Cartan connexion on $U$ iff there are piecewise smooth functions $\gamma_{j k}^{i}$ in $A\left(\mathrm{St} u_{j} \cap \mathrm{S} t u_{k}\right)$, for each $i, j$, and $k$, for which

$$
D\left(d u_{i}\right)=\sum_{j, k} \gamma_{j k}^{i} d u_{k} \otimes d u_{j}
$$

and for which $\gamma_{j k}^{i}=0$ on $\mathscr{C}\left(\operatorname{St} u_{i}\right) \cap \operatorname{St} u_{j} \cap \operatorname{St} u_{k}$.

Proof. Given $D$, the existence of the $\gamma_{j k}^{i}$ is obvious. The fact that $\gamma_{\jmath k}^{i}=0$ on $\mathscr{C}\left(\mathrm{St} u_{i}\right) \cap \mathrm{St} u_{j} \cap \mathrm{St} u_{k}$ follows since $D$ is an $A(U)$ derivation.

Conversely, given $\gamma_{j k}^{i}$ in $A\left(\mathrm{St} u_{j} \cap \mathrm{St} u_{k}\right)$ for which $\gamma_{j k}^{i}=0$ on $\mathscr{C}\left(\mathrm{St} u_{i}\right) \cap \mathrm{St} u_{j} \cap \mathrm{St} u_{k}$, define $D$ by

$$
\begin{aligned}
D\left(\sum_{i} f_{i} d u_{i}\right) & =D\left(\sum_{i} \bar{f}_{i} \cdot d u_{i}\right) \\
& =\sum_{i}\left(d \bar{f}_{i} \otimes d u_{i}+\bar{f}_{i} D\left(d u_{i}\right)\right) \\
& =\sum_{i}\left(d \bar{f}_{i} \otimes d u_{i}+\bar{f}_{i}\left(\sum_{j, k} \gamma_{j k}^{i} d u_{k} \otimes d u_{j}\right)\right)
\end{aligned}
$$

where $\bar{f}_{i}=f_{i}$ if St $u_{i}=U$, and $\bar{f}_{i} \in A(U)$ is an extension of $f_{i} \in A\left(\operatorname{St} u_{i}\right)$ 
otherwise. To show that this makes sense, first observe that if $\theta \in E(U)$ and $\theta=0$ on St $u_{i}$, then $\theta \otimes d u_{i} \in E(U) \otimes E(U)$ is 0 . (If $\theta=\sum_{j} g_{j} d u_{j}$, then $\theta=0$ on St $u_{i}$ implies that either $u_{j \mid \mathrm{St} u_{i} \cap \mathrm{St} u_{j}} \neq 0$, in which case $g_{j}=0$ on St $u_{i} \cap \mathrm{St} u_{j}$, or $u_{\left.j \mid \mathrm{St} u_{i}\right\urcorner \mathrm{St} u_{j}}=0$. In the first case

$$
g_{j \mid \mathrm{St} u_{i} \cap \mathrm{St} u_{j}} d u_{j} \otimes d u_{i}=0,
$$

and in the latter $d u_{j} \otimes d u_{i}=0$.) Thus given extensions $\bar{f}_{i}^{\prime}$ and $\bar{f}_{i}^{\prime \prime}$ of $f_{i}, d \bar{f}_{i}^{\prime}-d \bar{f}_{i}^{\prime \prime}=0$ on St $u_{i}$ so that

$$
d \bar{f}_{i}^{\prime} \otimes d u_{i}-d \bar{f}_{i}^{\prime \prime} \otimes d u_{i}=\left(d \bar{f}_{i}^{\prime}-d \bar{f}_{i}^{\prime \prime}\right) \otimes d u_{i}=0 .
$$

Second observe that since $\bar{f}_{i}$ is well defined on $u_{i}$ and $\gamma_{j k}^{i}=0$ on $\mathscr{C}\left(\mathrm{St} u_{i}\right) \cap \mathrm{St} u_{j} \cup \mathrm{St} u_{k}$, clearly $\left(\bar{f}_{i \mid \mathrm{St} u_{j} \cap \mathrm{St} u_{k}}\right) \gamma_{j k}^{i}$ is well defined on St $u_{j} \cap$ St $u_{k}$. Since this is the support of $d u_{k} \otimes d u_{j}, \bar{f}_{i} D\left(d u_{i}\right)$ is independent of the extension $\bar{f}_{i}$ of $f_{i}$.

CoRollary 4.5. Cartan connexions exist on $M$.

Proof. Let $\left\{\varphi_{i}\right\}_{i \in I}$ be a piecewise smooth partition of unity subordinate to a locally finite cover $\left\{U_{i}\right\}_{i \in I}$ of $M$ by small open subsets. For each $i \in I$, define the Cartan connexion

$$
D_{i}: E\left(U_{i}\right) \longrightarrow E\left(U_{i}\right) \otimes E\left(U_{i}\right)
$$

on $U_{i}$ by letting $D_{i}\left(d u_{j}\right)=0$ for each $j$. It is easy to verify that the map

$$
D: E(M) \longrightarrow E(M) \otimes E(M)
$$

given by $D \theta=\sum_{i} \varphi_{i} D_{i}\left(\left.\theta\right|_{U_{i}}\right)$ is a Cartan connexion on $M$.

We now describe connexions on $M$ by continuous vector fields.

Definition 4.6. A Koszul connexion on $M$ is a real-bilinear map

$$
\begin{aligned}
\nabla: \mathscr{X}(M) \times \mathscr{X}(M) & \longrightarrow \mathscr{X}(M) \\
(X, Y) & \longmapsto \nabla_{X} Y
\end{aligned}
$$

such that $\nabla_{f X} Y=f \nabla_{X} Y$ and $\nabla_{X} f Y=X(f) Y+f \nabla_{X} Y$ for $f \in A(M)$ and $X, Y \in \mathscr{X}(M)$.

Again, as in the smooth case, a Koszul connexion $\nabla$ on $M$ determines a Koszul connexion

$$
\nabla_{U}: \mathscr{X}(U) \times \mathscr{X}(U) \longrightarrow \mathscr{X}(U)
$$

for every small open subset $U \subseteq M$.

THEOREM 4.7. Let $U \subseteq M$ be small open neighborhood of $x_{0}$, let $u=\left\{u_{1}, \cdots, u_{N}\right\}$ be a coordinate system on $U$ with origin $x_{0}$ for which 
$\left\{u_{1}, \cdots, u_{p}\right\}$ coordinatizes $U_{\alpha}=\sigma_{\alpha} \cap U$ where $x_{0}$ is contained in the interior of the $p$-simplex $\sigma_{\alpha} \subseteq M$, and let

$$
X_{i}= \begin{cases}\partial / \partial u_{i} & \text { for } \quad i=1, \cdots, p \\ u_{i}\left(\partial / \partial u_{i}\right) & \text { for } \quad i=p+1, \cdots, N .\end{cases}
$$

Then

$$
\nabla: \mathscr{P}(U) \times \mathscr{X}(U) \longrightarrow \mathscr{X}(U)
$$

is a Koszul connexion on $U$ iff there are piecewise smooth functions $\delta_{j k}^{i}$ in $A\left(\right.$ St $\left.u_{i}\right)$, for each $i, j$, and $k$, for which

$$
\nabla_{X_{j}} X_{k}=\sum_{i} \delta_{j k}^{i} X_{i}
$$

and for which $\delta_{j k}^{i}=0$ on St $u_{i} \cap \mathscr{C}\left(\operatorname{St} u_{j} \cap \operatorname{St} u_{k}\right)$.

Proof. Given $\nabla$, the existence of the $\delta_{j k}^{i}$ is obvious. The fact that $\delta_{j k}^{i}=0$ on $\mathrm{St} u_{i} \cap \mathscr{C}\left(\mathrm{St} u_{j} \cap \mathrm{St} u_{k}\right.$ ) follows since $\nabla_{X} Y$ is $A(U)$ linear in $X$ and an $A(U)$-derivation in $Y$.

Conversely, given $\delta_{j k}^{i}$ in $A\left(\mathrm{St} u_{i}\right)$ for which $\delta^{i}{ }_{j k}=0$ on St $u_{i} \cap$ $\mathscr{C}\left(\mathrm{St} u_{j} \cap \mathrm{St} u_{k}\right)$, we first let

$$
\nabla_{f X_{j}} X_{k}=\nabla_{\bar{f} X_{j}} X_{k}=\bar{f} \nabla_{X_{j}} X_{k}=\bar{f}\left(\sum_{\imath} \delta_{j k}^{i} X_{i}\right)
$$

where $\bar{f}=f$ if St $u_{j}=U$, and $\bar{f} \in A(U)$ is an extension of $f \in A\left(\operatorname{St} u_{j}\right)$ otherwise. This makes sense since $\bar{f}$ is well defined on St $u_{j}$ and $\delta_{j k}^{i}=0$ on St $u_{i} \cap \mathscr{C}\left(\right.$ St $u_{j} \cap$ St $\left.u_{k}\right)$ so that $\bar{f} \delta_{j k}^{i} \in A\left(\right.$ St $\left.u_{i}\right)$ is well defined.

Next define

$$
\begin{aligned}
\nabla_{X_{j}} f X_{k} & =\nabla_{X_{j}} \bar{f} X_{k} \\
& =X_{j}(\bar{f}) X_{k}+\bar{f} \nabla_{X_{j}} X_{k} \\
& =X_{j}(\bar{f}) X_{k}+\bar{f}\left(\sum_{i} \delta_{j k}^{i} X_{i}\right)
\end{aligned}
$$

where $\bar{f}=f$ if St $u_{k}=U$, and $\bar{f} \in A(U)$ is an extension of $f \in A\left(\right.$ St $\left.u_{k}\right)$ otherwise. To see that this makes sense, first notice that since $X_{j}$ is a derivation, $f=0$ on St $u_{i}$ implies that $X_{j}(f)=0$ on St $u_{i}$, so that $X_{j}(f) X_{i}=0$ on $U$; thus given extensions $\bar{f}_{i}^{\prime}$ and $\bar{f}_{i}^{\prime \prime}$ of $f_{i}$,

$$
X_{j}\left(\bar{f}_{i}^{\prime}\right) X_{\imath}-X_{j}\left(\bar{f}_{i}^{\prime \prime}\right) X_{i}=X_{j}\left(\bar{f}_{i}^{\prime}-\bar{f}_{i}^{\prime \prime}\right) X_{i}=0
$$

since $\bar{f}_{i}^{\prime}-\bar{f}_{i}^{\prime \prime}=0$ on St $u_{i}$. Second observe that $\bar{f}\left(\sum_{i} \delta_{j k}^{i} X_{i}\right)$ is well defined; this is precisely the same argument as above for $\bar{f} \delta_{j k}^{i}$.

Finally define $\nabla$ on all of $\mathscr{X}(U) \times \mathscr{X}(U)$ by extending by reallinearity. 
TheOREM 4.8. There is a bijection between the set of Cartan connexions on $M$ and the set of Koszul connexions on $M$.

Proof. It is not difficult to show that for every $X \in \mathscr{X}(M)$ there is a real-linear map

$$
\operatorname{eval}_{X}: E(M) \otimes E(M) \longrightarrow E(M)
$$

which is given locally, on a small open subset $U \subseteq M$, by

$$
\operatorname{eval}_{X}\left(\sum_{i} \theta_{i} \otimes \varphi_{i}\right)=\sum_{i}\left\langle X, \varphi_{i}\right\rangle \theta_{i}
$$

where " $\langle-,-\rangle$ " denotes "evaluation" (see Theorem 3.5). One now defines the bijection between Cartan connexions $D$ on $M$ and Koszul connexions $\nabla$ on $M$ by imposing the contraction formula

$$
\left\langle\nabla_{X} Y, \theta\right\rangle=X\langle Y, \theta\rangle+\left\langle Y, \operatorname{eval}_{X} D \theta\right\rangle
$$

where $X, Y \in \mathscr{Q}(M)$ and $\theta \in E(M)$.

To describe the correspondence of Theorem 4.8 locally, let $U$ be a small open neighborhood of $x_{0} \in M$, let $u=\left\{u_{1}, \cdots, u_{N}\right\}$ be a coordinate system on $U$ with origin $x_{0}$ such that $\left\{u_{1}, \cdots, u_{p}\right\}$ coordinatizes $U_{\alpha}=\sigma_{\alpha} \cap U$ where $x_{0}$ is contained in the interior of the $p$-simplex $\sigma_{\alpha} \subseteq M$, and let

$$
X_{i}= \begin{cases}\partial / \partial u_{i} & \text { for } \quad i=1, \cdots, p \\ u_{i}\left(\partial / \partial u_{i}\right) & \text { for } \quad i=p+1, \cdots, N .\end{cases}
$$

If the Cartan connexion $D$ on $U$ given by $D\left(d u_{i}\right)=\sum_{j, k} \gamma_{j k}^{i} d u_{k} \otimes d u_{j}$ and the Koszul connexion $\nabla$ on $U$ given by $\nabla_{X_{j}} X_{k}=\sum_{i} \delta_{j k}^{i} X_{i}$ correspond to each other via Theorem 4.8, then the relations between $\gamma_{j k}^{j}$ and $\delta_{j k}^{i}$ are given by:

if $i=$

$1, \cdots, p$

$1, \cdots, p$

$1, \cdots, p$

$1, \cdots, p$

$p+1, \cdots, N 1, \cdots, p$

$p+1, \cdots, N \quad 1, \cdots, p$

$p+1, \cdots, N p+1, \cdots, N$

$p+1, \cdots, N \quad p+1, \cdots, N \quad p+1, \cdots, N \begin{cases}u_{i} \delta_{j k}^{i}=u_{j} u_{k} \gamma_{j k}^{i} & \text { if } i \neq j \\ & \text { or } i \neq k \\ \delta_{j k}^{i}=1+u_{i} \gamma_{j k}^{i} & \text { if } i=j=k .\end{cases}$

if $k=\quad$ relation

$$
1, \cdots, p \quad \delta_{j k}^{i}=\gamma_{j k}^{2}
$$$$
p+1, \cdots, N \delta_{j k}^{i}=u_{k} \gamma_{j k}^{i}
$$

$1, \cdots, p$

$\delta_{j k}^{i}=u_{j} \gamma_{j k}^{i}$

$p+1, \cdots, N \delta_{j k}^{i}=u_{j} u_{k} \gamma_{j k}^{i}$

$1, \cdots, p \quad u_{i} \delta_{j k}^{i}=\gamma_{j k}^{i}$

$p+1, \cdots, N \quad u_{i} \delta_{j k}^{i}=u_{k} \gamma_{j k}^{i}$

$1, \cdots, p$

$u_{i} \delta_{j k}^{i}=u_{j} \gamma_{j k}^{i}$$$
u_{i} \delta_{j k}=u_{j} \gamma_{j k}
$$ 
The following result is an immediate consequence of Theorem 4.8 and Corollary 4.5.

COROllary 4.9. Koszul connexions exist on $M$.

It is easy to show that if $\nabla$ is a Koszul connexion on $M$ then for every $x_{0} \in M$ and $X, Y \in \mathscr{X}(M)$, the value of $\nabla_{X} Y$ at $x_{0}$ depends only on $X_{x_{0}}$ and the values of $Y$ on some curve that fits $X_{x_{0}}$.

THEOREM 4.10. A Koszul connexion $\nabla$ on $M$ is equivalent to a collection

$$
\nabla_{\alpha}: \mathscr{X}\left(\sigma_{\alpha}\right) \times \mathscr{X}\left(\sigma_{\alpha}\right) \longrightarrow \mathscr{X}\left(\sigma_{\alpha}\right)
$$

of Koszul connexions $\nabla_{\alpha}$ defined on the simplices $\sigma_{\alpha} \leqq M$ which are compatible in the following sense: If $\sigma_{\alpha}, \sigma_{\beta} \subseteq M$ are simplices for which $\sigma_{\alpha} \subseteq \sigma_{\beta}, x_{0}$ is in the interior of $\sigma_{\alpha}, X \in T_{x_{0}} \sigma_{\alpha}$, and $Y$ is a smooth vector field on some smooth curve in $\sigma_{\alpha}$ which fits $X$, then

$$
\left(D_{\alpha}\right)_{X} Y=\left(D_{\beta}\right)_{\left(i_{\beta \alpha}\right) * X}\left(i_{\beta \alpha}\right)_{*} Y,
$$

$\left(i_{\beta \alpha}\right)_{*}$ denoting the Jacobian of the inclusion $i_{\beta \alpha}: \sigma_{\alpha} \rightarrow \sigma_{\beta}$.

Proof. Given a Koszul connexion $\nabla$ on $M$, let $U$ be a small open neighborhood of $x_{0} \in M$ and let $u=\left\{u_{i}\right\}$ be a coordinate system on $U$ with origin $x_{0}$. For each wedge $U_{\alpha} \subseteq U$, let $u_{\alpha}=\left\{u_{\alpha}^{i}\right\}$ denote the coordinate system on $U_{\alpha}$ induced by $u$. If

$$
D: E(M) \longrightarrow E(M) \otimes E(M)
$$

is the Cartan connexion on $M$ associated to $\nabla$ and

$$
D: E(U) \longrightarrow E(U) \otimes E(U)
$$

is the induced Cartan connexion on $U$, given in coordinate form by

$$
D\left(d u_{i}\right)=\sum_{j, k} \gamma_{j k}^{i} d u_{k} \otimes d u_{j},
$$

then for every wedge $U_{\alpha} \subseteq U$ there is a unique Koszul connexion

$$
\nabla_{\alpha}: \mathscr{X}\left(U_{\alpha}\right) \times \mathscr{C}\left(U_{\alpha}\right) \longrightarrow \mathscr{X}\left(U_{\alpha}\right)
$$

given by

$$
\left(\nabla_{\alpha}\right)_{\partial / \partial u_{\alpha}^{j}} \partial / \partial u_{\alpha}^{k}=\sum_{i}\left(\gamma_{j k \mid U_{\alpha}}^{i}\right)\left(\partial / \partial u_{\alpha}^{i}\right) .
$$

This construction is natural, and hence a Koszul connexion

$$
\nabla_{\alpha}: \mathscr{X}\left(\sigma_{\alpha}\right) \times \mathscr{X}\left(\sigma_{\alpha}\right) \longrightarrow \mathscr{X}\left(\sigma_{\alpha}\right)
$$


is defined on each simplex $\sigma_{\alpha} \subseteq M$. The compatibility of these connexions is immediate.

Conversely, given a compatible collection of connexions as described in the hypothesis, let $X, Y \in \mathscr{Z}(M)$. To define $D_{X} Y$ at $x_{0} \in M$, let $\sigma_{\alpha} \subseteq M$ be the simplex in whose interior $x_{0}$ is contained. By Corollary 2.5 there are smooth vector fields $X_{\alpha}, Y_{\alpha} \in \mathscr{P}\left(\sigma_{\alpha}\right)$ for which $\left(i_{\alpha}\right)_{*} X_{\alpha}=$ $X$ and $\left(i_{\alpha}\right)_{*} Y_{\alpha}=Y$ on $\sigma_{\alpha}$. There is clearly a smooth curve in $\sigma_{\alpha}$ which fits $X_{x_{0}}$, and since $Y_{\alpha}$ is defined along this curve we may let

$$
\left(\nabla_{X} Y\right)_{x_{0}}=\left(i_{\alpha}\right)_{*}\left(D_{\alpha}\right)_{\left(X_{\left.\alpha^{\prime}\right)} x_{0}\right.} Y_{\alpha} .
$$

This clearly defines a Koszul connexion on $M$ and the proof is complete.

We will next use Theorem 4.10 to interpret connexions geometrically. First, however, recall (see [7]) that a piecewise smooth curve $f:[a, b] \rightarrow M$ in $M$ is a map for which there is a finite subdivision

$$
a=c_{0}<c_{1}<\cdots<c_{N}=b
$$

of $[a, b]$ such that for each $i=0, \cdots, N-1$ there is a simplex $\sigma_{\alpha} \subseteq M$ for which $f:\left[c_{i}, c_{i+1}\right] \rightarrow \sigma_{\alpha}$ is a smooth curve in $\sigma_{\alpha}$.

DEFINITION 4.11. A continuous vector field along a piecewise smooth curve $f:[a, b] \rightarrow M$ is a map $Y:[a, b] \rightarrow T M$ which is smooth on each subinterval of the subdivision $\left({ }^{*}\right)$ of $[a, b]$ and for which $\tau(M) \circ Y=f$.

Now let $\nabla$ be a Koszul connexion on $M$. Let $\sigma_{\alpha}, \sigma_{\beta} \subseteq M$ be $n$ simplices whose intersection $\sigma_{\gamma}$ is a $p$-simplex of $M$, and let $\nabla_{\alpha}$ and $\nabla_{\beta}$ be the (compatible) connexions on $\sigma_{\alpha}$ and $\sigma_{\beta}$ induced by $\nabla$. Finally let $f:[a, b] \rightarrow M$ be a piecewise smooth curve for which there is a $c \in(a, b)$ such that

$$
\begin{aligned}
& f_{\alpha}=\left.f\right|_{[a, c]}:[a, c] \longrightarrow \sigma_{\alpha}, \\
& f_{\beta}=\left.f\right|_{[c, b]}:[c, b] \longrightarrow \sigma_{\beta},
\end{aligned}
$$

are smooth curves in $\sigma_{\alpha}$ and $\sigma_{\beta}$, respectively.

TheOREM 4.12. For each $t \in[a, b]$ there is a p-dimensional subspace $V_{t} \subseteq T_{f(t)} M$ such that for every $Y_{0} \in V_{a}$ there is a continuous vector field $Y=Y_{t}$ on $f$ for which $Y_{t} \in V_{t}$ for each $t \in[a, b], Y_{a}=Y_{0}, Y_{\alpha}=$ $\left.Y\right|_{[a, c]}$ is parallel along $f_{\alpha}$ with respect to $\nabla_{\alpha}$, and $Y_{\beta}=\left.Y\right|_{[c, b]}$ is parallel along $f_{\beta}$ with respect to $\nabla_{\beta}$.

Proof. With respect to $\nabla_{\alpha}$, for every $Y_{0} \in T_{f(a)} \sigma_{\alpha}$ there is a unique smooth vector field $Y_{\alpha}=\left(Y_{\alpha}\right)_{t}$ on $\sigma_{\alpha}$ such that $\left(Y_{\alpha}\right)_{0}=Y_{0}$ and $Y_{\alpha}$ is 
parallel along $f_{\alpha}$, and a linear isomorphism

$$
P_{a, t}^{\alpha}: T_{f(a)} \longrightarrow T_{f(t)}
$$

called parallel translation along $f_{a}$ from $f(a)$ to $f(t)$; similarly for $\sigma_{\beta}$. With the notation of Corollary 2.5, for $t \in[a, c]$ we let

$$
V_{t}=\left(i_{\alpha}\right)_{*}\left(P_{t, c}^{\alpha}\right)^{-1}\left(i_{\alpha \gamma}\right)_{*} T_{f(c)} \sigma_{\gamma} \subseteq T_{f(t)} M
$$

and for $t \in[c, b]$ we let

$$
V_{t}=\left(i_{\beta}\right)_{*}\left(P_{c, t}^{\beta}\right)\left(i_{\beta \gamma}\right)_{*} T_{f(c)} \sigma_{\gamma} \subseteq T_{f(t)} M ;
$$

observe that $V_{t}$ is well defined for $t=c$. For $Y_{0} \in V_{a}$, we define $Y=Y_{t}$ on $f$ by

$$
Y_{t}= \begin{cases}\left(i_{\alpha}\right)_{*}\left(P_{a, t}^{\alpha}\right)\left(i_{\alpha}\right)_{*}^{-1} Y_{0} & \text { for } t \in[a, c] \\ \left(i_{\beta}\right)_{*}\left(P_{c, t}^{\beta}\right)\left(i_{\beta}\right)_{*}^{-1}\left(i_{\alpha}\right)_{*}\left(P_{a, c}^{\alpha}\right)\left(i_{\alpha}\right)_{*}^{-1} Y_{0} & \text { for } t \in[c, b] .\end{cases}
$$

Observe that $P_{a, t}$ is independent of $c$ since $\nabla_{\alpha}$ and $\nabla_{\beta}$ are compatible.

Thus there is a map $P_{a, t}: V_{a} \rightarrow V_{t}$ defined by $P_{a, t}\left(Y_{0}\right)=Y_{t}$ which is a linear isomorphism and which can reasonably be called parallel translation along $f$ from $f(a)$ to $f(t)$.

Theorem 4.12 is still valid under the hypothesis that $\sigma_{\alpha}, \sigma_{\beta} \subseteq M$ are simplices of arbitrary dimension whose intersection is a $p$-simplex $\sigma_{\gamma}$ of $M$.

\section{REFERENCES}

1. S. Cairns, Triangulated manifolds which are not Brouwer manifolds, Annals of Math., 41 (1940), 792-795.

2. N. Hicks, Notes on Differential Geometry, Van Nostrand Reinhold Co., New York, 1965.

3. C. D. Marshal, Calculus on subcartesian spaces, J. Differential Geometry, 10 (1975), $551-574$.

4. H. Osborn, Differential Geometry in PL, (mimeographed notes), University of Illinois, Urbana-Champaign, 1971.

5. - Function algebras and the de Rham theorem in PL, Bull. Amer. Math. Soc., 77 (1971), 386-391.

6. M. Penna, Differential Geometry on Simplicial Spaces, Trans. Amer. Math. Soc., 214 (1975), 303-323.

7. - Vector Fields on Polyhedra, to appear in Trans. Amer. Math. Soc.

8. M. Spivak, $A$ Comprehensive Introduction to Differential Geometry, Publish or Perish, Boston, 1970.

9. E. C. Zeeman, Polyhedral N-Manifolds: I. Foundations, pp. 57-64 of Topology of 3-Manifolds, Prentice-Hall, Englewood Cliffs, New Jersey, 1962.

Received May 12, 1977.

INDIANA UNIVERSITY-PURDUE UNIVERSITY AT INDIANAPOLIS

INDIANAPOLIS, IN 46205 


\title{
PACIFIC JOURNAL OF MATHEMATICS
}

\section{EDITORS}

RICHARD ARENS (Managing Editor)

University of California

Los Angeles, CA 90024

Charles W. Curtis

University of Oregon

Eugene, OR 97403

C. C. MOORE

University of California

Berkeley, CA 94720
J. DUGUNDJI

Department of Mathematics

University of Southern California

Los Angeles, CA 90007

R. Finn and J. Milgram

Stanford University

Stanford, CA 94305

\section{ASSOCIATE EDITORS}
E. F. BECKENBACH
B. H. Neumann
F. WOLF
K. YosHidA

\section{SUPPORTING INSTITUTIONS}

\author{
UNIVERSITY OF BRITISH COLUMBIA \\ CALIFORNIA INSTITUTE OF TECHNOLOGY \\ UNIVERSITY OF CALIFORNIA \\ MONTANA STATE UNIVERSITY \\ UNIVERSITY OF NEVADA, RENO \\ NEW MEXICO STATE UNIVERSITY \\ OREGON STATE UNIVERSITY \\ UNIVERSITY OF OREGON
}

\author{
UNIVERSITY OF SOUTHERN CALIFORNIA \\ STANFORD UNIVERSITY \\ UNIVERSITY OF HAWAII \\ UNIVERSITY OF TOKYO \\ UNIVERSITY OF UTAH \\ WASHINGTON STATE UNIVERSITY \\ UNIVERSITY OF WASHINGTON
}

The Supporting Institutions listed above contribute to the cost of publication of this Journal, but they are not owners or publishers and have no responsibility for its content or policies.

Mathematical papers intended for publication in the Pacific Journal of Mathematics should be in typed form or offset-reproduced, (not dittoed), double spaced with large margins. Please do not use built up fractions in the text of the manuscript. However, you may use them in the displayed equations. Underline Greek letters in red, German in green, and script in blue. The first paragraph or two must be capable of being used separately as a synopsis of the entire paper. Items of the bibliography should not be cited there unless absolutely necessary, in which case they must be identified by author and journal, rather than by item number. Manuscripts, in triplicate, may be sent to any one of the editors. Please classify according to the scheme of Math. Reviews, Index to Vol. 39. All other communications should be addressed to the managing editor, or Elaine Barth, University of California, Los Angeles, California, 90024.

50 reprints to each author are provided free for each article, only if page charges have been substantially paid. Additional copies may be obtained at cost in multiples of 50 .

The Pacific Journal of Mathematics is issued monthly as of January 1966. Regular subscription rate: $\$ 72.00$ a year (6 Vols., 12 issues). Special rate: $\$ 36.00$ a year to individual members of supporting institutions.

Subscriptions, orders for numbers issued in the last three calendar years, and changes of address should be sent to Pacific Journal of Mathematics, P.O. Box 969, Carmel Valley, CA 93924, U.S.A. Older back numbers obtainable from Kraus Periodicals Co., Route 100, Millwood, NY 10546.

PUBLISHED BY PACIFIC JOURNAL OF MATHEMATICS, A NON-PROFIT CORPORATION

Printed at Kokusai Bunken Insatsusha (International Academic Printing Co., Ltd.). 8-8, 3-chome, Takadanobaba, Shinjuku-ku, Tokyo 160, Japan.

Copyright (C) 1978 by Pacific Journal of Mathematics Manufactured and first issued in Japan 


\section{Pacific Journal of Mathematics}

Vol. 77, No. $2 \quad$ February, 1978

Graham Donald Allen, Duals of Lorentz spaces ................... 287

Gert Einar Torsten Almkvist, The number of nonfree components in the decomposition of symmetric powers in characteristic p .......... 293

John J. Buoni and Bhushan L. Wadhwa, On joint numerical ranges ...... 303

Joseph Eugene Collison, Central moments for arithmetic functions . . . . . . 307

Michael Walter Davis, Smooth G-manifolds as collections of fiber

bundles ........................................ 315

Michael E. Detlefsen, Symmetric sublattices of a Noether lattice......... 365

David Downing, Surjectivity results for $\phi$-accretive set-valued

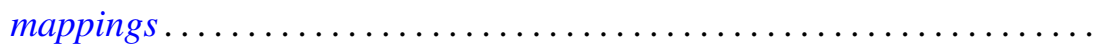

David Allyn Drake and Dieter Jungnickel, Klingenberg structures and partial designs. II. Regularity and uniformity ................. 389

Edward George Effros and Jonathan Rosenberg, $C^{*}$-algebras with approximately inner flip .......................... 417

Burton I. Fein, Minimal splitting fields for group representations. II. . . . . 445

Benjamin Rigler Halpern, A general coincidence theory ............. 451

Masamitsu Mori, A vanishing theorem for the mod $p$ Massey-Peterson spectral sequence ................................ 473

John C. Oxtoby and Vidhu S. Prasad, Homeomorphic measures in the

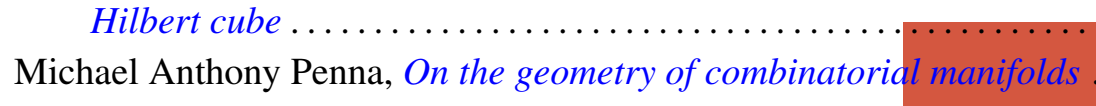

Robert Ralph Phelps, Gaussian null sets and differentiability of Lipschitz map on Banach spaces........................

Herbert Silverman, Evelyn Marie Silvia and D. N. Telage, Locally univalent functions and coefficient distortions...

Donald Curtis Taylor, The strong bidual of $\Gamma(K)$

Willie Taylor, On the oscillatory and asymptotic behavior of solutions of fifth order selfadjoint differential equations ...........

Fu-Chien Tzung, Sufficient conditions for the set of Hausdorff compactifications to be a lattice. 